\title{
Ellipsoids are the only local maximizers of the volume product
}

\author{
Mathieu Meyer and Shlomo Reisner
}

\begin{abstract}
Using previous results about shadow systems and Steiner symmetrization, we prove that the local maximizers of the volume product of convex bodies are actually the global maximizers, that is: ellipsoids.
\end{abstract}

Let $K \subset \mathbb{R}^{n}$ be a convex body (a compact and convex set with non-empty interior). For $z \in \operatorname{int}(K)$, the interior of $K$, let $K^{z}$ be the polar of $K$ with respect to $z$ :

$$
K^{z}=\left\{y \in \mathbb{R}^{n} ;\langle y-z, x-z\rangle \leq 1 \text { for every } x \in K\right\},
$$

where $\langle.,$.$\rangle denotes the standard scalar product in \mathbb{R}^{n}$. It is well known that $K^{z}$ is also a convex body, that $z \in \operatorname{int}\left(K^{z}\right)$ and that $\left(K^{z}\right)^{z}=K$. The volume product of $K$, $\Pi(K)$ (or $\Pi_{n}(K)$ if the dimension is to be specified), is given by the following formula:

$$
\Pi(K):=\min _{z \in \operatorname{int}(K)}|K|\left|K^{z}\right|,
$$

where $|A|$ denotes the Lebesgue measure of a Borel subset $A$ of $\mathbb{R}^{n}$. The unique point $z=s(K) \in K$, where this minimum is reached, is called the Santaló point of $K$. We denote $K^{*}=K^{s(K)}$. Blaschke [B] (1917) proved for dimensions $n=2$ and $n=3$ that

$$
\Pi(K)=|K|\left|K^{*}\right| \leq \Pi\left(B_{2}^{n}\right),
$$

where $B_{2}^{n}=\left\{x \in \mathbb{R}^{n} ;|x| \leq 1\right\}(|x|=\sqrt{\langle x, x\rangle})$ is the Euclidean unit ball in $\mathbb{R}^{n}$. This was generalized to all dimensions by Santaló [San] (1948).

It then took some time to establish the case of equality: one has $\Pi(K)=\Pi\left(B_{2}^{n}\right)$ if and only if $K$ is an ellipsoid. This was done by Saint-Raymond [Sai] (1981), when $K$ is centrally symmetric and by Petty $[\mathrm{P}]$ (1982), in the general case. Another proof was given by Meyer and Pajor [MP] (1990), based on Steiner symmetrization.

2010 Mathematics Subject Classification 52A20, 52A40.

Key words and phrases: convex bodies, volume, volume-product, Blaschke-Santaló inequality. 
Campi and Gronchi [CG] (2006), introduced the use of shadow systems for volume product problems. Fix a direction $u \in S^{n-1}$. A shadow system $\left(K_{t}\right)$ along the direction $u$ is a family of convex sets $\left(K_{t}\right), t \in[a, b]$ such that

$$
K_{t}=\operatorname{conv}\{x+t \alpha(x) u ; x \in A\}
$$

where $A$ is a given bounded subset of $\mathbb{R}^{n}$ and $\alpha: A \rightarrow \mathbb{R}$ ia a given bounded function, called the speed of the shadow system. An example is given by the Steiner symmetrization of a convex body $K$ with respect to the hyperplane $u^{\perp}$ orthogonal to $u \in S^{n-1}$. If $K$ is described as

$$
K=\left\{y+s u ; y \in P_{u} K, s \in I(y)\right\},
$$

where $P_{u}$ is the orthogonal projection onto $u^{\perp}$ and $I(y)$ is some nonempty closed interval depending on $y \in P_{u} K$. The Steiner symmetral $\mathrm{St}_{u}(K)$ is defined by

$$
\mathrm{St}_{u}(K):=\left\{y+s u ; y \in P_{u} K, s \in \frac{I(y)-I(y)}{2}\right\} .
$$

For $t \in[-1,1]$, let

$$
K_{t}=\left\{y+s u ; y \in P_{u} K, s \in \frac{1-t}{2} I(y)-\frac{1+t}{2} I(y)\right\} .
$$

The family $\left(K_{t}\right), t \in[-1,1]$ forms a shadow system such that $K_{-1}=K, K_{1}$ is the reflection of $K$ with respect to $u^{\perp}$ and $K_{0}$ is the Steiner symmetral of $K$ with respect to $u^{\perp}$. As a matter of fact, setting $A=K_{0}$, and $I(y)=[a(y), b(y)]$ for $y \in P_{u} K$, one has for $t \in[-1,1]$ :

$$
K_{t}=\left\{z-t \frac{a\left(P_{u} z\right)+b\left(P_{u} z\right)}{2} u ; z \in K_{0}\right\} .
$$

The following theorem was proved in [MR2] as Theorem 1 and Proposition 7 there.

Theorem 1 Let $K_{t}, t \in[a, b]$, be a shadow system in $\mathbb{R}^{n}$. Then $t \rightarrow\left|K_{t}^{*}\right|^{-1}$ is a convex function on $[a, b]$. If $t \rightarrow\left|K_{t}\right|$ and $t \rightarrow\left|K_{t}^{*}\right|^{-1}$ are both affine functions in $[a, b]$ then, for all $t \in[a, b], K_{t}$ is an affine image of $K_{a}, K_{t}=A_{u, t}\left(K_{a}\right)$. Where $A_{u, t}$ is an affine transformation that satisfies $P_{u} A_{u, t}=P_{u}$. More precisely: for some $v \in \mathbb{R}^{n}$ and some $c \in \mathbb{R}$, one has for all $t \in[-1,1]$ and all $x \in \mathbb{R}^{n}$ :

$$
A_{u, t}(x)=x+(t-a)(\langle x, v\rangle+c) u .
$$

This theorem was extending and strengthening a result of Campi and Gronchi [CG], who proved the first part of it when the shadow system $\left(K_{t}\right)$ is composed of bodies that are centrally symmetric with respect to the same center of symmetry.

As a consequence of Theorem 1, one gets the main result of this paper:

Theorem 2 The convex bodies $K$ in $\mathbb{R}^{n}$ which are local maximizers (with respect to the Hausdorff distance or to the Banach Mazur distance) of the volume product in $\mathbb{R}^{n}$ are the ellipsoids. 
Remark. A partial result in this direction was proved by Alexander, Fradelizi and Zvavich [AFZ] who observed that no polytope can be a local maximizer for the volume product.

Proof of Theorem 2. Suppose that $K$ is a local maximizer. Let $u \in S^{n-1}$ and $\mathrm{S} t_{u}(K)$ be the Steiner symmetral of $K$ with respect to $u^{\perp}$.

With the above notations we describe the Steiner symmetral of $K$ as $K_{0}$ of a shadow system $K_{t}, t \in[-1,1]$, with $K_{-1}=K$ and $K_{1}$ being the mirror reflection of $K$ about $u^{\perp}$. It follows from the definition of this shadow system that it preserves the volume of $K$ : one has $\left|K_{t}\right|=|K|$ for all $t \in[-1,1]$.

By construction, for all $t, K_{t}$ is the mirror reflection of $K_{-t}$ with respect to $u^{\perp}$. It follows that $\left(K_{t}\right)^{*}$ is also the mirror reflection of $\left(K_{-t}\right)^{*}$ with respect to $u^{\perp}$ Let

$$
f(t)=\left(|K|\left|\left(K_{t}\right)^{*}\right|\right)^{-1}=\frac{1}{\Pi_{n}\left(K_{t}\right)} .
$$

It is clear that the function $t \rightarrow K_{t}$ is continuous for both the Hausdorff and the Banach-Mazur distances. Thus such is also the function $t \rightarrow\left(K_{t}\right)^{*}$. It follows that $f$ is continuous on $[-1,1]$.

By theorem 1, $f$ is convex on $[-1,1]$ and by construction, it is even. Thus $f(t) \leq$ $f(-1)=f(1)$ for all $t \in[-1,1]$ and $f$ has its absolute minimum at 0 . Since $K$ is a local maximum of the volume product (i.e, a local minimum of $f$ ), one has for some $-1<c \leq 0, f(t) \geq f(-1)$ for all $t \in[-1, c]$. Thus $f$ is constant on $[-1, c]$. It now follows from its convexity and the preceding observations, that $f$ is actually constant on $[-1,1]$ and $\left|\left(K_{t}\right)^{*}\right|=\left|K^{*}\right|$ for $t \in[-1,1]$.

From the second part of theorem 1 we conclude now that $K_{0}=\mathrm{St}_{u}(K)$ is an image of $K_{-1}=K$ under an affine transformation having special properties. Since this fact is true for any $u \in S^{n-1}$, application of the next lemma completes the proof.

Lemma 3 Let $K$ be a convex body such that, for all $u \in S^{n-1}$, $\operatorname{St}_{u}(K)$ is an image of $K, S t_{u}(K)=A_{u}(K)$ where $A_{u}$ is an affine transformation that satisfies $P_{u} A_{u}=P_{u}$. Then (and only then) $K$ is an ellipsoid.

Remark. Lemma 3 can be formulated in an equivalent form as: Let $K$ be a convex body such that, for all $u \in S^{n-1}$, the centers of the chords of $K$ that are parallel to $u$ are located on a hyperplane. Then (and only then) $K$ is an ellipsoid. With this formulation the result, in dimension 2, was declared by Bertrand [Ber] (1842). But his proof does not seem complete. The result was proved by Brunn [Br] (1889). Gruber [Gr] (1974) proved the result under strongly relaxed assumptions. A number of proofs of the result appear in the literature. See e.g. Danzer, Laugwitz and Lenz [DLL] (1957), that use the Löwner ellipsoid of $K$, or Grinberg Gri] (1991) that uses an infinite sequence of symmetrizations. We bring here, for the sake of completeness, a proof that uses the uniqueness of the John ellipsoid of $K$.

We also point out [MR1] for a generalization, replacing the location of midpoints of chords by the location of centroids of sections of any fixed dimension $k, 1 \leq k \leq n-1$.

Proof of Lemma 3. We notice that the property of $K$ presented in the lemma is preserved under affine transformations (this is easy to see from the equivalent form of 
this property presented in the Remark above). Thus, using an affine transformation, we may assume that John's ellipsoid of $K$ (the ellipsoid of maximal volume contained in $K$ ) is the Euclidean unit ball $B_{2}^{n}$. We then want to show that $K$ is a homothetic Euclidean ball.

Let $u \in S^{n-1}$. By the assumption, $S t_{u}(K)=A_{u}(K), A_{u}$ affine with $P_{u} A_{u}=P_{u}$. Hence the John ellipsoid of $S t_{u}(K)$ is $A_{u}\left(B_{2}^{n}\right)$. Now $|K|=\left|S t_{u}(K)\right|=\left|A_{u}(K)\right|$, so $\left|\operatorname{det}\left(A_{u}\right)\right|=1$ and $\left|A_{u}\left(B_{2}^{n}\right)\right|=\left|B_{2}^{n}\right|$. By symmetry of $B_{2}^{n}$ about $u^{\perp}$ and the fact that $B_{2}^{n} \subset K$, we have $B_{2}^{n} \subset S t_{u}(K)$. By the uniqueness of the John ellipsoid we conclude that $A_{u}\left(B_{2}^{n}\right)=B_{2}^{n}$. Thus $A_{u}$ is a linear isometry with respect to the Euclidean norm, i.e. an orthogonal transformation.

The orthogonal transformation $A_{u}$ preserves $u^{\perp}$ by the assumption of the lemma, so it is either the identity or an orthogonal reflection by $u^{\perp}$. Using any of these possibilities for each $u \in S^{n-1}$, we see that $K$ is orthogonally symmetric about any hyperplane through 0 . It follows that all the points of the boundary of $K$ have the same Euclidean norm. Thus $K$ is a Euclidean ball centered at the origin.

This completes the proof of Lemma 3 , thus also the proof of Theorem 2 ,

\section{References}

[AFZ] M. Alexander, M. Fradelizi And A. Zvavitch, Polytopes of Maximal Volume Product, arXiv:1708.07914 (2017).

[Ber] J. Bertrand, Démonstration d'un théorème de géométrie, Journal de Mathématiques Pures et Appliquées 7 (1842), 215-216.

[B] W. Blaschke, Über affine Geometrie VII: Neue Extremeingenschaften von Ellipse und Ellipsoid, Ber. Verh. Sächs. Akad. Wiss., Math. Phys. Kl. 69 (1917), 412-420.

[Br] H. BRunN, Über Kurven ohne Wendepunkte, Habilitationsschrift, Munich (1889).

[CG] S. CAmpi And P. Gronchi, On volume product inequalities for convex sets, Proc. Amer. Math. Soc. 134 (2006), 2393-2402.

[DLL] L. Danzer, D. Laugwitz And H. Lenz, Über das Lównersche Ellipsoid und sein analogon unter den einem Eikörper einbeschriebenen Ellipsoiden, Arch. Math. 8 (1957), 214-219.

[Gri] E. L. Grinberg, Isoperimetric inequalities and identities for $k$-dimensional cross-sections of convex bodies, Math. Ann. 291 (1991), 75-86.

[Gr] P. GRUBER, Über kennzeichende Eigenschaften von Euklidischen Räumen und Ellipsoiden I, J. für die reine und angewandte Mathematik 265 (1974), 61-83.

[MP] M. Meyer And A. PAjor, On the Blaschke-Santaló inequality, Arch. Math. (Basel) 55 (1990), no. 1, 82-93. 
[MR1] M. MEyer AND S. REISNER, Characterizations of ellipsoids by sectioncentroid location, Geometriae Dedicata 31 (1989), 345-355.

[MR2] M. MEYER AND S. REISNER, Shadow systems and volumes of polar convex bodies, Mathematika 53 (2006), no. 1, 129-148.

[P] C. M. Petty, Affine isoperimetric problems, Discrete geometry and convexity, Ann. New York Acad. Sci., vol. 440 (New-York 2002), New York Acad. Sci., New York, 1985, pp. 113-127.

[Sai] J. SAInt Raymond, Sur le volume des corps convexes symétriques, Séminaire d'Initiation à l'Analyse: G. Choquet-M. Rogalski-J. Saint-Raymond, 20ème Année: 1980/1981, Publ. Math. Univ. Pierre et Marie Curie, vol. 46, Univ. Paris VI, Paris, 1981, pp. Exp. No. 11, 25.

[San] L. A. Santaló, Un invariante afin para los cuerpos convexos del espacio des n dimensiones, Portugaliae Math. 8 (1949), 155-161.

M. Meyer: Université Paris-Est - Marne-la-Vallée, Laboratoire d'Analyse et de Mathématiques Appliquées (UMR 8050), Cité Descartes, 5 Bd Descartes, Champs-sur-Marne, 77454 Marnela-Vallée cedex 2, France.

Email: mathieu.meyer@u-pem.fr

S. Reisner: Department of Mathematics, University of Haifa, Haifa 31905, Israel.

Email: reisner@math.haifa.ac.il 